\title{
BMJ Open Protocol for a prospective cohort study of assessing postoperative cognitive changes after total hip and knee arthroplasty in the Greater Toronto area
}

\author{
Stephen Choi, Sinziana Avramescu, Beverley A Orser, Shelly Au
}

To cite: Choi S, Avramescu S, Orser BA, et al. Protocol for a prospective cohort study of assessing postoperative cognitive changes after total hip and knee arthroplasty in the Greater Toronto area. BMJ Open 2019;9:e024259. doi:10.1136/ bmjopen-2018-024259

- Prepublication history and additional material for this paper are available online. To view these files, please visit the journal online (http://dx.doi org/10.1136/bmjopen-2018024259).

Received 17 May 2018 Revised 20 December 2018 Accepted 7 January 2019

\section{Check for updates}

(c) Author(s) (or their employer(s)) 2019. Re-use permitted under CC BY-NC. No commercial re-use. See rights and permissions. Published by BMJ.

Department of Anesthesiology, Sunnybrook Health Sciences Center, Toronto, Ontario, Canada

Correspondence to Dr Stephen Choi; stephen.choi@sunnybrook.ca

\section{ABSTRACT}

Introduction Cognitive changes after anaesthesia and surgery, such as delirium and postoperative cognitive dysfunction (POCD), are common and lead to poor outcomes and increased healthcare costs. While several interventions for delirium exist, there are no effective treatment strategies for POCD. Understanding the risks and contributing factors may offer clinicians unique opportunities to better identify and develop preventative interventions for those at higher risk. Elderly patients undergoing orthopaedic surgery are at high risk of developing postoperative delirium (PD) and POCD. The incidence of POCD has not been rigorously studied in the total hip and knee arthroplasty (THATTKA) population. Therefore, we have designed a prospective, observational cohort study to assess POCD in patients undergoing THATTKA, both increasingly common procedures. The incidence of $\mathrm{PD}$ and POCD in a high volume, tertiary care arthroplasty centre will be determined and associated risk factors will be identified. Methods and analysis Cognitive function will be tested with a computer-based cognitive assessment tool [CogState Brief Battery], preoperatively at baseline and postoperatively while in hospital at ( $<3$ days), 6 weeks and 4.5 months. The primary outcome is the incidence of postoperative cognitive decline at 4.5 months. Logistic regression analysis is planned to test the association of POCD with several potential risk factors. In addition, delirium will be assessed preoperatively and postoperatively in the hospital using the Confusion Assessment Method (3D-CAM).

Ethics and dissemination The protocol for this prospective observational study was approved by the Sunnybrook Health Sciences Centre Research Ethics Board (REB\#: 040-2017). Recruitment commenced in May 2017 and will continue until 2019. The results will be disseminated in a peer-reviewed journal and in scientific meetings.

Trial registration number NCT03147937.

\section{INTRODUCTION}

Cognitive changes after anaesthesia and surgery are frequent and often lead to poor outcomes and increased healthcare costs. Postoperative delirium (PD) is an acute, fluctuating change in cognitive function that affects up to $53 \%$ of patients after surgery. Postoperative cognitive dysfunction (POCD)
Strengths and limitation of the study

Observational, non-randomised design only examining association, not causation.

- Examines potential risk factors previously not investigated in the total hip and knee arthroplasty (THA/ TKA) population.

- Assesses the total THA/TKA population, a procedure performed with increasing frequency.

- Rapid, repeatable cognitive assessment of participants whose performance is compared with age/ sex-matched controls from a large worldwide sample.

- Does not establish cognitive trajectories prior to surgical procedure.

is a persistent deterioration of cognition that lasts well beyond the expected effects of anaesthesia and surgery. For the purposes of this trial, POCD is defined as cognitive deterioration lasting longer than 3 months. Recommendations have recently been published for changes to POCD nomenclature to align the term 'cognitive dysfunction' with the diagnostic terminology used in the DSM-5 (ie, neurocognitive disorder); however, the traditional nomenclature will be utilised for the remainder of this work as acceptance of the new terminology is not yet widespread. ${ }^{2}$

Several seminal studies have demonstrated varying degrees of POCD. The International Study of Postoperative Cognitive Dysfunction (ISPOCD) in 1998, replicated by Monk et al in 2008, demonstrated respectively that $9.9 \%$ and $10 \%$ of patients suffered from POCD 3 months after a major, non-cardiac surgery. ${ }^{34}$ The International Study of Postoperative Cognitive Dysfunction -2 (ISPOCD2) study demonstrated POCD in $14.3 \%$ of patients after general anaesthesia and $13.9 \%$ after regional anaesthesia at 3 months. ${ }^{5}$ At 1 year, Ballard et al demonstrated $11.2 \%$ of patients had POCD (compared with $3.8 \%$ in non-surgical controls) while Abildstrom 
demonstrated a similar incidence of $10.4 \%$, and McDonagh et al found that $46.1 \%$ of individuals had POCD after elective, non-cardiac surgery. ${ }^{6-8}$ Both PD and POCD have been associated with increased morbidity, mortality, loss of independence and increased healthcare costs. ${ }^{49}{ }^{10}$ Each episode of PD is estimated to increase hospital costs between $\$ 16000$ and \$64 000. ${ }^{9}$ With approximately 300 million surgical procedures (approximately one third in those $>65$ years) performed worldwide annually, the costs associated with PD are enormous. ${ }^{11}$ This does not include costs associated with long-term care for patients with POCD. They are also distressing to patients and their families. While there are several non-pharmacological interventions that help reduce the incidence of $\mathrm{PD},{ }^{12}$ there are no effective strategies for preventing or treating POCD. Given the high incidence and poor outcomes, understanding the risk factors is crucial in the development of effective preventative interventions for POCD.

Elderly patients undergoing orthopaedic surgery are at higher risk of developing PD and POCD. ${ }^{3413}$ The incidence of POCD after major elective orthopaedic surgery at 1 year was nearly threefold higher $(11.2 \%$ vs $3.8 \%)$ than in age-matched non-surgical controls. ${ }^{6}$ The incidence of POCD after major joint arthroplasty has not been rigorously studied. However, these are among the most commonly performed surgical procedures in the USA with 700000 knee replacements and 300000 hip replacements performed in $2010 .{ }^{14}$ These numbers have approximately doubled since 2000 and are expected to continue to grow. Therefore, patients should be cognizant of the potential negative impact they may have on their cognitive function. Several studies on POCD in elective arthroplasty or emergent hip fracture surgery utilise cognitive screens such as the Mini-Mental Status Examination or Montreal Cognitive Assessment, neither of which is intended to assess cognition in depth while only assessing 1-week postoperatively. ${ }^{15-19}$ The time-frame examined and cognitive tests utilised are suboptimal.

Therefore, we designed a study to determine the incidence of negative cognitive outcomes (POCD at 4.5 months) in elderly patients undergoing elective total THA/TKA utilising the computerised CogState Brief Battery (CBB). Furthermore, we aim to assess whether factors such as mild cognitive impairment (MCI), chronic inflammatory states, $\mathrm{PD}$ and postoperative complications, demonstrated to have negative effects on cognition in the non-operative population are associated with POCD. ${ }^{20-23}$ This study will allow us to establish a research platform that utilises high throughput validated tools to assess cognitive function in the perioperative period which can be used to assess the efficacy of novel interventions in future trials to either prevent or treat POCD.

\section{METHODS}

\section{Study design}

We will perform a single-centre, prospective, observational cohort study. All approved amendments to this

Box 1 Inclusion and exclusion criteria

Inclusion criteria

$>250$ years of age.

- Elective unilateral total hip or knee arthroplasty.

\section{Exclusion criteria}

- Lack of informed consent.

- Inability to comply with study procedures or follow-up visits.

- Diagnosed dementia or being treated with donepezil.

- Preoperative delirium (positive 3D-CAM).

- Severe cognitive impairment defined as baseline CogState Brief Battery (CBB) score of $\leq 80$ in at least one of the four CBB domains.

- Uncontrolled psychiatric diagnoses including schizophrenia, bipolar disorder and major depressive mood disorder.

Previous enrolment in this study within the past 6 months.

study will be communicated to relevant parties. Study end points will be assessed via in-person computerised tests during the participants' routine postoperative follow-up visits with their orthopaedic surgeon, review of patient medical records and telephonic interviews.

\section{Study population and setting}

The study sample will be drawn from patients that present for THA/TKA to the Holland Orthopaedic and Arthritic Centre (HOAC) of Sunnybrook Health Sciences Centre (SHSC), a tertiary-care academic health sciences centre affiliated with the University of Toronto. The HOAC is an orthopaedic centre of excellence and is the highest volume THA/TKA centre in Canada with approximately 3000 procedures performed annually. All procedures are elective and all patients are evaluated in the preoperative anaesthesia clinic approximately 2 weeks before surgery. The surgical procedure and anaesthetic technique are standardised. Patients are discharged home or to a rehabilitation facility between postoperative days 2 and 4 with standardised care pathways. There is an opportunity for careful follow-up with patients returning for regularly scheduled postoperative appointments at defined intervals ( 6 weeks \pm 2 weeks and 4.5 months \pm 6 weeks).

\section{Recruitment and study assessments}

Study inclusion and exclusion criteria are listed in box 1 . Patients scheduled for unilateral TKA or THA at our institution will be approached for participation in the preoperative assessment clinic ( 2-4 weeks prior to surgery) as outlined in the study assessment schedule (figure 1). Research personnel will follow good clinical practice and ensure that participants have a complete understanding of the study prior to obtaining their written consent. In addition to completing a baseline demographic review of the patients' medical history, all consenting participants will complete the 3D-CAM and CBB prior to surgery (baseline).

Patients will receive standard care, both intraoperatively and postoperatively, with no changes due to study participation. Postoperatively, patients will be assessed for delirium (3D-CAM) and changes in their cognitive 


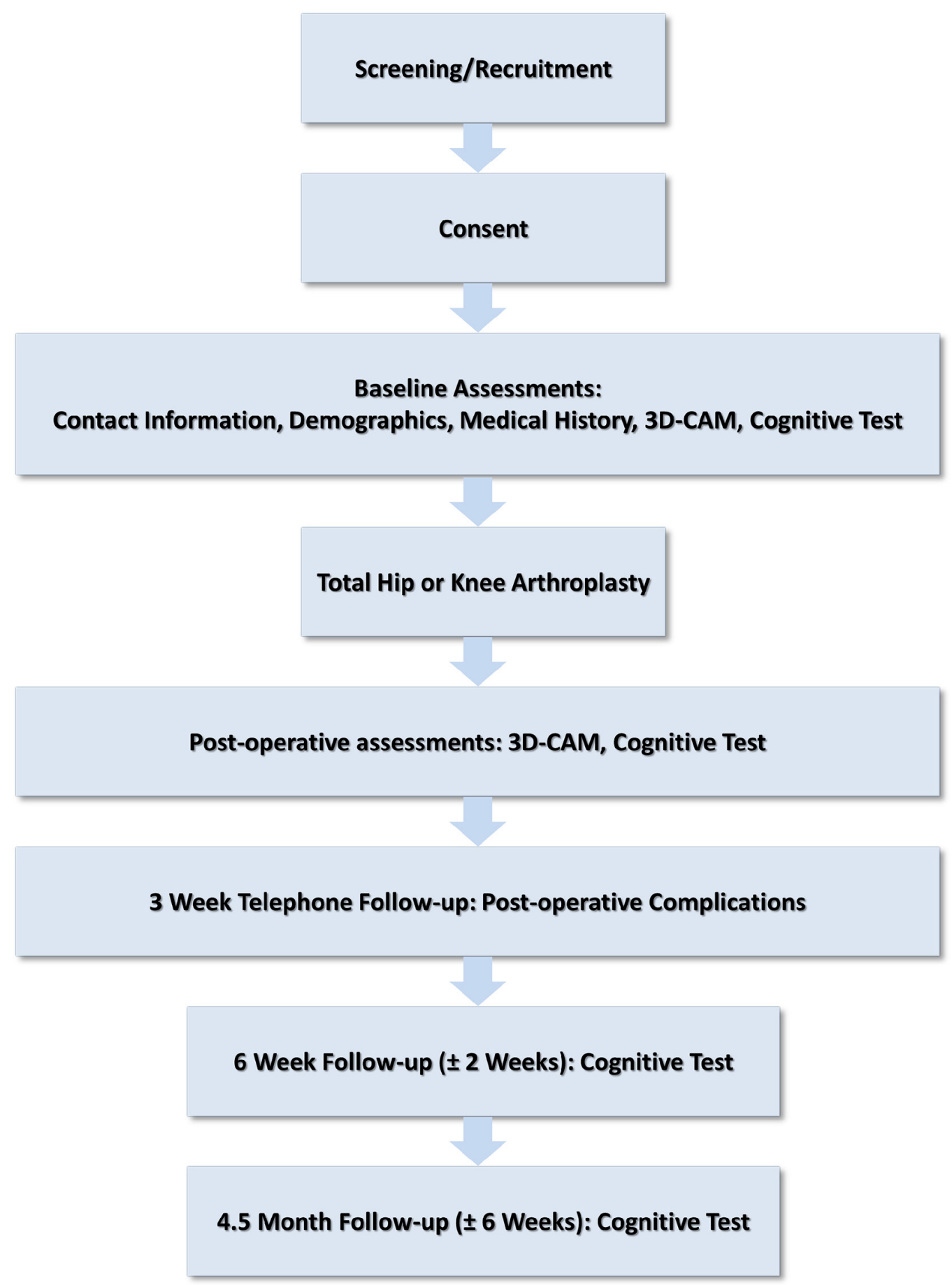

Figure 1 Study flowchart of patients consenting and enrolled in the study.

function (CBB). Postoperative complications and total opioid consumption during the first three postoperative days will be recorded.

Participants are scheduled to return for postoperative follow-ups with their surgeons at approximately 6 weeks $( \pm 2$ weeks) and 4.5 months ( \pm 6 weeks) after surgery. Participants will be contacted by telephone 3 weeks after surgery to confirm follow-up visit dates and inquire about the occurrence of complications. At each in-person follow-up visit, $\mathrm{CBB}$ will be administered and inquiries regarding the occurrence of complications will occur.
Relevant medical records will be obtained in the event of any identified postoperative complications.

\section{CogState Brief Battery}

The CBB is based on a standard deck of 52 playing cards (spades, clubs, diamonds and hearts from 2 to ace) and validated against commonly employed neuropsychological tests ${ }^{2425}$ and in the perioperative period. ${ }^{2627}$ It assesses the four cognitive domains (psychomotor function, attention, learning and memory, and working memory) employed in POCD research, first used by Moller et al 
(ISPOCD group) and replicated by Monk et al. ${ }^{34}$ It does have some advantages over traditional neuropsychological tests, including ease of administration, shorter testing time allowing for repeated high-volume testing, lack of language or cultural barriers and the lack of a learning effect from repeated administration. Furthermore, test scores are compared with a large, worldwide database of age and sex-matched controls, maximising the validity of the test and repeated measurements can document chronological changes to model cognitive trajectories. Use of the CBB addresses concerns from previous POCD research and is novel in the approach of comparison to controls drawn from a worldwide population rather than small numbers of healthy controls. ${ }^{28}$

\section{Anaesthetic management}

The standard anaesthetic technique is spinal anaesthesia with isobaric $0.5 \%$ bupivacaine $(7.5-15 \mathrm{mg}$ depending on anticipated surgical duration) combined with fentanyl (10-15ug). Approximately $95 \%$ of patients undergoing THA/TKA at HOAC undergo spinal anaesthesia with supplemental sedation (propofol infusion titrated to a Ramsay Sedation Scale score of 3 or 4). A small proportion undergoing general anaesthesia do so for several reasons including patient preference, medical issues precluding spinal anaesthesia or inability to access the intrathecal space. Current data suggests that anaesthetic modality does not influence the incidence of POCD. ${ }^{29} 30$

\section{Postoperative analgesia}

Patients undergoing THA receive postoperative multimodal analgesia with acetaminophen and non-steroidal anti-inflammatories, where not contraindicated, as well as opioids on demand. For patients undergoing TKA, in addition to multimodal analgesia, continuous femoral nerve block with a local anaesthetic (ropivacaine $0.15 \%$ $5-7 \mathrm{~mL} /$ hour) for 2 days postoperatively is utilised.

\section{Outcomes}

The primary outcome of this prospective trial is the incidence of POCD at 4.5 months, defined as a CBB score of $<80$ in any one of the four tasks. This corresponds to a cognitive function in one of the four domains that is $>2$ SD lower than age and sex-matched controls. This time point was chosen for the convenience of patients as they routinely complete a follow-up visit with their surgeon at 4.5 months. The secondary outcome will be the incidence of patients developing MCI at 4.5 months, defined as a CBB score between 81 and 90 corresponding to a decline in cognitive function of 1 to 2 SDs compared with age and sex-matched controls. All data being collected are listed in box 2.

\section{Data analysis}

A logistic regression model will be used to determine the association between POCD at 4.5 months and the following variables: presence of preoperative chronic inflammatory state (online supplementary appendix 1 ), preoperative MCI, PD, presence of central nervous

\section{Box 2 Data collected for each patient}

The following preoperative data will be collected from each patient on enrolment into the study:

- Age (coded by decile: 50-59, 60-69, 70-79, 80-89,>90).

- Sex (Male/Female).

- American Society of Anesthesiologists' Physical Stauts (ASA) classification.

- Surgical procedure.

- Highest level of education attained (lower than high school, high school, some undergraduate, undergraduate degree, postgraduate degree).

- Presence/absence of preoperative delirium (PD).

- Pre-existing central nervous system diagnosis (eg, Parkinson's, previous stroke, multiple sclerosis).

- Pre-existing auto-immune disease (eg, rheumatoid arthritis, systemic lupus erythematosus).

- Pre-existing chronic disease associated with inflammation (eg, coronary artery disease, obstructive sleep apnea).

- Pre-existing chronic pain defined by preoperative opioid consumption of $>30 \mathrm{mg}$ oral morphine equivalent (opioid conversion as per Canadian Compendium of Pharmaceuticals).

- Preoperative mild cognitive impairment (at least two or more out of four cognitive testing domains with a score between 81-90).

The following data will be collected from patients
postoperatively:
Acute postoperative complications assessed at each postoperative
follow-up visit (eg, cardiac event, pulmonary embolus, respiratory
failure, pneumonia, prosthetic joint infection).
CogState Brief Battery score on Postoperative Day (POD) 1,2 or 3 and
first and second postoperative follow-up visits ( 6 weeks $+/-2$ weeks
and 4.5 months +/- 6 weeks).
Presence/absence of PD.
Anaesthetic modality - general anaesthesia vs spinal anaesthesia.
Length of stay.

system pathology (online supplementary appendix 1), postoperative complications (online supplementary appendix 1) and highest educational status attained. The same variables, apart from preoperative MCI, will be used in a logistic regression model to estimate the effect of each variable on the development of postoperative MCI. While age and sex have previously been identified as risk factors, they are not included in the analysis as the CBB normalises subjects' test scores to age and sex-matched controls. Analyses will be performed using SPSS Statistical Software, V. 20 (IBM, Armonk, NY, USA). P values will be reported to 3 decimal places. For all inferential tests, the type I error level will be set at 0.05 .

\section{Sample size estimation}

The sample size estimated is based on the reported incidence of POCD 3 months after major elective surgery of approximately $10 \%$. A minimum of 10 events per variable is necessary to adequately produce estimates of effect with regression models. ${ }^{31}{ }^{32}$ A sample size of 600 individuals (it is estimated that 60 will develop POCD) will allow six variables to be assessed in the regression model. 


\section{ETHICS AND DISSEMINATION}

Participants will not experience any undue hardship, expenses, or time commitments because the test sequence (CBB) will be carried out during the participants' routine preoperative and postoperative visits as well as during the inpatient period. Personal health information and cognitive assessment results will be kept confidential. Measures to maintain security include transposition of initials with a confidential algorithm and using false birth dates but maintaining actual month and year of birth. The month and year of birth are required to allow for comparison with age-matched controls.

Knowledge translation of the results of this study will be accomplished through publication in peer-reviewed journals as well as presentation at international and local conferences, meetings, events, workshops and symposiums. Equally important stakeholders including public health officials, orthopaedic surgeons and patients will have to be included as there is potential for significant impact in both of these fields over and beyond that of anaesthesiology.

\section{PATIENT AND PUBLIC INVOLVEMENT}

This specific project was not initiated at the request of a particular patient, but the avenue of investigation (ie, POCD) was prompted by questions/concerns brought forward by patients and their families to the investigators. Individual patient level feedback was utilised to introduce measures in the study that would facilitate follow-up but they were not specifically involved in the design or recruitment/conduct of the study.

\section{DISCUSSION}

SHSC performs approximately 3000 THA/TKA per year, primarily under spinal anaesthesia. Utilising our retrospective data, the exclusion criteria would further reduce the eligible sample population to approximately 2400 yearly. We have recently conducted a pilot study to assess recruitment and the feasibility of recruiting the full sample size. While the feasibility threshold was to recruit 15 participants per month, our pilot study recruited 95 patients over 3 months. The data from the 95 patients of the pilot study will be utilised, requiring a further 505 participants to be recruited to reach the full sample size of 600 study participants completing the 4.5 month follow-up. We expect that more than 600 individuals will be required to participate to enable 600 successful completions of the CBB at 4.5 months.

This study aims to address several of the methodological limitations identified in POCD literature. ${ }^{33}$ These include not accounting for natural cognitive decline associated with ageing, the negative cognitive effects of pre-existing comorbid conditions (eg, cardiovascular or metabolic diseases), the negative cognitive effects of postoperative critical illness/complications, lack of a consensus definition of POCD, adjustment of test scores because of learning effects from repeated cognitive testing and not establishing preoperative cognitive trajectories. While the study design and assessment selection attempts to address several concerns, not all are addressed. CBB accounts for ageing as participants' scores are compared with age and sex-matched controls from the general population; and because it is based on standard playing cards, there is a near infinite number of alternate forms obviating the need for adjustments due to learning effects. Furthermore, the study analysis attempts to compensate for external factors (premorbid conditions and postoperative complications) that may influence cognition. With respect to consensus definitions, the criteria used to define MCI and POCD are based on the recently published consensus statement recommendations regarding the nomenclature of postoperative cognitive changes. ${ }^{2}$ It does not, however, establish preoperative trajectories. Establishing long-term preoperative trajectories will be the most challenging aspect of any prospective POCD study.

There are several other limitations of this study. Primarily, it is an observational study and therefore susceptible to unequal distribution of prognostic variables. Second, POCD is not designated as an entity in the International Classification of Disease and therefore, diagnostic criteria that have been reported in many other studies are variable. Nonetheless, there are individuals who dispute the existence of persistent POCD. CBB will help provide an easy-to-communicate, repeatable, validated measure of cognitive function after surgery that will allow repeated assessments to follow change and potentially establish a framework in which POCD can be better defined. Additionally, while the POCD literature typically assessed study participants at 1 week, 3 months, and at times 1 year, our study will be assessing participants in hospital (2 days), at 6 weeks and 4.5 months. This schedule coincides with regular follow-up and was made to enhance participant recruitment/follow-up while minimising participant burden but will complicate comparing the results to the existing literature. Finally, there will be inevitable loss to follow-up due to perioperative mortality that has an incidence of $1 \%$ following major orthopaedic surgery and loss to follow-up from participant fatigue and scheduling issues.

It is hoped that this study, by using CBB and incorporating preoperative and postoperative risk factors for cognitive decline, will provide a more accurate assessment of postoperative cognitive changes after THA/TKA. The study of this area is particularly germane, given the push towards fast-track surgery and strategies to minimise the likelihood or magnitude of POCD can then be developed to alter this major public health issue. ${ }^{34}$

Acknowledgements We would like to extend our gratitude to all study participants for their contributions.

Contributors SC designed study protocol, obtained funding, and is involved with study conduct, data analysis; SA obtained funding, and is involved with study conduct; BAO obtained funding; SA wrote first draft of manuscript, 
and is involved with study conduct. All authors edited and approved the final manuscript.

Funding This work is supported by the Sunnybrook Academic Health Sciences Centre Alternative Funding Plan Innovation Fund. The investigators received an in-kind donation for no cost access to the CBB from CogState Ltd. Funders have no role in the study design, data collection, management, analysis or interpretation of the data.

Disclaimer The views, statements and opinions presented in this work are solely the responsibility of the authors. CogState Limited did not have a role in the development of the protocol or analysis plan. Furthermore, they have no role in data analysis or dissemination of results.

Competing interests None declared.

Patient consent for publication Not required.

Provenance and peer review Not commissioned; externally peer reviewed.

Open access This is an open access article distributed in accordance with the Creative Commons Attribution Non Commercial (CC BY-NC 4.0) license, which permits others to distribute, remix, adapt, build upon this work non-commercially, and license their derivative works on different terms, provided the original work is properly cited, appropriate credit is given, any changes made indicated, and the use is non-commercial. See: http://creativecommons.org/licenses/by-nc/4.0/.

\section{REFERENCES}

1. Inouye SK. Delirium in older persons. N Engl J Med 2006;354:1157-65

2. Evered L, Silbert B, Knopman DS, et al. Recommendations for the nomenclature of cognitive change associated with anaesthesia and surgery-2018. Br J Anaesth 2018;121:1005-12.

3. Moller JT, Cluitmans P, Rasmussen LS, et al. Long-term postoperative cognitive dysfunction in the elderly ISPOCD1 study. ISPOCD investigators. International Study of Post-Operative Cognitive Dysfunction. Lancet 1998;351:857-61.

4. Monk TG, Weldon BC, Garvan CW, et al. Predictors of cognitive dysfunction after major noncardiac surgery. Anesthesiology 2008:108:18-30.

5. Rasmussen LS, Johnson T, Kuipers HM, et al. Does anaesthesia cause postoperative cognitive dysfunction? A randomised study of regional versus general anaesthesia in 438 elderly patients. Acta Anaesthesiol Scand 2003;47:260-6.

6. Ballard C, Jones E, Gauge N, et al. Optimised anaesthesia to reduce post operative cognitive decline $(P O C D)$ in older patients undergoing elective surgery, a randomised controlled trial. PLOS One 2012;7:e37410.

7. McDonagh DL, Mathew JP, White WD, et al. Cognitive function after major noncardiac surgery, apolipoprotein E4 genotype, and biomarkers of brain injury. Anesthesiology 2010;112:852-9.

8. Abildstrom H, Rasmussen LS, Rentowl P, et al. Cognitive dysfunction 1-2 years after non-cardiac surgery in the elderly. ISPOCD group. International Study of Post-Operative Cognitive Dysfunction. Acta Anaesthesiol Scand 2000;44:1246-51.

9. Leslie DL, Marcantonio ER, Zhang Y, et al. One-year health care costs associated with delirium in the elderly population. Arch Intern Med 2008;168:27-32.

10. Steinmetz J, Christensen KB, Lund T, et al. Long-term consequences of postoperative cognitive dysfunction. Anesthesiology 2009;110:548-55.

11. Weiser TG, Haynes AB, Molina G, et al. Size and distribution of the global volume of surgery in 2012. Bull World Health Organ 2016;94:201-9.

12. Inouye SK, Bogardus ST, Charpentier PA, et al. A multicomponent intervention to prevent delirium in hospitalized older patients. N Engl J Med 1999;340:669-76.

13. Contín AM, Perez-Jara J, Alonso-Contín A, et al. Postoperative delirium after elective orthopedic surgery. Int $J$ Geriatr Psychiatry 2005;20:595-7.
14. Centers for Disease Prevention and Control. National Hospital Discharge Survey: 2010 table. 2014 http://www.cdc.gov/nchs/ fastats/inpatient-surgery.htm

15. Sun S, Sun D, Yang L, et al. Dose-dependent effects of intravenous methoxamine infusion during hip-joint replacement surgery on postoperative cognitive dysfunction and blood TNF- $\alpha$ level in elderly patients: a randomized controlled trial. BMC Anesthesiol 2017;17:75

16. Mei B, Meng G, Xu G, et al. Intraoperative sedation with dexmedetomidine is superior to propofol for elderly patients undergoing hip arthroplasty: a prospective randomized controlled study. Clin J Pain 2018;34:811-7.

17. Fan Y, Yuan L, Ji M, et al. The effect of melatonin on early postoperative cognitive decline in elderly patients undergoing hip arthroplasty: A randomized controlled trial. J Clin Anesth 2017;39:77-81.

18. Wu XM, Xu WC, Yu YJ, et al. Postoperative serum thioredoxin concentrations correlate with delirium and cognitive dysfunction after hip fracture surgery in elderly patients. Clin Chim Acta 2017;466:93-7.

19. Papadopoulos G, Karanikolas M, Liarmakopoulou A, et al. Cerebral oximetry and cognitive dysfunction in elderly patients undergoing surgery for hip fractures: a prospective observational study. Open Orthop J 2012;6:400-5.

20. Ancelin ML, de Roquefeuil G, Scali J, et al. Long-term post-operative cognitive decline in the elderly: the effects of anesthesia type, apolipoprotein E genotype, and clinical antecedents. J Alzheimers Dis 2010;22 Suppl 3(s3):S105-S113.

21. Silbert BS, Scott DA, Evered LA, et al. Preexisting cognitive impairment in patients scheduled for elective coronary artery bypass graft surgery. Anesth Analg 2007;104:1023-8.

22. Terrando N, Monaco C, Ma D, et al. Tumor necrosis factor-alpha triggers a cytokine cascade yielding postoperative cognitive decline. Proc Natl Acad Sci U S A 2010;107:20518-22.

23. Veliz-Reissmüller $\mathrm{G}$, Agüero Torres $\mathrm{H}$, van der Linden J, et al. Pre-operative mild cognitive dysfunction predicts risk for postoperative delirium after elective cardiac surgery. Aging Clin Exp Res 2007:19:172-7.

24. Darby DG, Pietrzak RH, Fredrickson J, et al. Intraindividual cognitive decline using a brief computerized cognitive screening test. Alzheimers Dement 2012;8:95-104.

25. Maruff $P$, Thomas E, Cysique L, et al. Validity of the CogState brief battery: relationship to standardized tests and sensitivity to cognitive impairment in mild traumatic brain injury, schizophrenia, and AIDS dementia complex. Arch Clin Neuropsychol 2009;24:165-78.

26. Ichimura S, Ohira T, Kobayashi M, et al. Assessment of cognitive function before and after surgery for posterior cranial fossa lesions using computerized and conventional tests. Neurol Med Chir 2010;50:441-8

27. Silbert BS, Maruff P, Evered LA, et al. Detection of cognitive decline after coronary surgery: a comparison of computerized and conventional tests. Br J Anaesth 2004;92:814-20.

28. Newman S, Stygall J, Hirani S, et al. Postoperative Cognitive Dysfunction after Noncardiac Surgery. Anesthesiology 2007;106:572-90.

29. Evered L, Scott DA, Silbert B, et al. Postoperative cognitive dysfunction is independent of type of surgery and anesthetic. Anesth Analg 2011;112:1179-85.

30. Evered LA, Silbert BS, Scott DA, et al. Prevalence of dementia 7.5 years after coronary artery bypass graft surgery. Anesthesiology 2016;125:62-71.

31. Concato J, Peduzzi P, Holford TR, et al. Importance of events per independent variable in proportional hazards analysis. I. Background, goals, and general strategy. J Clin Epidemiol 1995;48:1495-501.

32. Peduzzi P, Concato J, Feinstein AR, et al. Importance of events per independent variable in proportional hazards regression analysis. II. Accuracy and precision of regression estimates. J Clin Epidemiol 1995;48:1503-10.

33. Nadelson MR, Sanders RD, Avidan MS. Perioperative cognitive trajectory in adults. Br J Anaesth 2014;112:440-51.

34. Vetter TR, Boudreaux AM, Jones KA, et al. The perioperative surgical home: how anesthesiology can collaboratively achieve and leverage the triple aim in health care. Anesth Analg 2014;118:1131-6. 